Les Cahiers du Gres

\section{Les nouvelles du Gres}

Volume 2, numéro 1, automne 2001

URI : https://id.erudit.org/iderudit/009425ar

DOI : https://doi.org/10.7202/009425ar

Aller au sommaire du numéro

\section{Éditeur(s)}

Groupe de recherche ethnicité et société

CEETUM

ISSN

1499-0431 (imprimé)

1499-044X (numérique)

Découvrir la revue

Citer ce document

(2001). Les nouvelles du Gres. Les Cahiers du Gres, 2(1), 85-86.

https://doi.org/10.7202/009425ar
Les Cahiers du Gres Série Diversité urbaine Groupe de Recherche Ethnicte et Sodetes 


\section{LES NOUVELLES DU GRES}

Le Groupe de Recherche Ethnicité et Société est heureux d'accueillir Linda Pietrantonio, une boursière post-doctorale (Conseil de Recherches en Sciences Humaines du Canada) pour l'année 2001. Les travaux de Madame Pietrantonio sur la notion d'égalité dans une perspective sociologique, et le développement de ceux-ci à partir d'une méthode d'analyse du discours social sont un apport important pour le GRES. En effet, s'étant récemment jointe au Groupe (janvier 2001), cette chercheure a déjà intégré et enrichi notre programme de recherche avec de nouveaux travaux qui s'insèrent de plain pied dans nos recherches actuelles qui traitent, de manière générale, de la dynamique interethnique de la société québécoise, telle qu'elle se présente dans la région montréalaise depuis les changements sociaux et politiques mis en oeuvre avec la Loi 101 (1977), visant la francisation de la vie publique.

Les chercheurs impliqués dans ce programme, comme membres de l'équipe ou comme collaborateurs sont Deirdre Meintel, Victor Piché, Danielle Juteau, Jean Renaud, Patricia Lamarre et Sylvie Fortin (professionnelle de recherche) de l'Université de Montréal ; Gladys Simons, de l'École nationale d'administration publique (Université du Québec); Marie Nathalie LeBlanc, de I'Université Concordia; Marguerite Cognet, du Centre de recherche et de formation du CLSC Côte-des-Neiges et Monica Heller, de l'Institut d'études pédagogiques de l'Ontario (Université de Toronto). Catherine Quiminal, de I'Unité de recherche Migration et société (URMIS), Université Paris 7 et Paris 8, collabore également aux travaux reliés à ce programme et participait, le printemps dernier, aux ateliers du GRES.

Les projets sont nombreux, les organismes subventionnaires étant aussi diversifiés ${ }^{1}$. Plusieurs assistants de recherche sont également mobilisés par ces projets, tels que Laurent Raigneau, Francine Lemire, Sébastien Arcand, Sirma Bilge, Sophie Ambrosi, Julie Paquette, Emmanuel Kahn, Hayley Wilson, Marco Guerrera et Josiane LeGall (coordonnatrice de projet), tous de I'Université de Montréal.

Le GRES est également très heureux d'accueillir, à la rentrée 2001, trois nouveaux stagiaires doctoraux, soient Alkistis Fleischer, Université de Georgetown (Washington, DC); Sabine Meyer, Université de Francfort-sur-leMain (Allemagne) et Paul Eid, Université de Toronto.

Mentionnons enfin trois nouvelles parutions des membres du GRES:

Les Cahiers du GRES, vol. 2, n¹, automne 2001 
Jean Renaud, Lucie Gingras, Sébastien Vachon, Christine Blaser, JeanFrançois Godin et Benoît Gagné. "Ils sont maintenant d'ici ! Les dix premières années au Québec des immigrants admis en 1989 ", Sainte Foy, Les publications du Québec, Collection Études, recherches et statistiques, 2001, 197 pages.

Bruno Ramirez, "Crossing the 49th Parallel. Migration from Canada to the United States, 1900-1930 » Cornell University Press, 2001.

Sylvie Fortin, « Destins et Défis. La migration libanaise à Montréal », Montréal, Les éditions Saint-Martin, 2000, 127 pages.

\section{Notes}

1 Ces organismes subventionnaires étant :

Fonds pour la Formation de Chercheurs et l'Aide à la Recherche (FCAR), Conseil de Recherches en Sciences Humaines du Canada (CRSH), Conseil Québécois de la Recherche Sociale (CQRS) et Patrimoine canadien, Multi-culturalisme Canada.

Les nouveaux projets subventionnés sont (printemps 2001) :

«Social Cohesion in a Linguistical Divided Society : Intergroup Relations among Montreal College Students », P. Lamarre, D. Meintel et M. Heller, Patrimoine Canada

«Prise de parole II: la francophonie canadienne et la nouvelle économie mondialisée ». D. Heller, A.T. Boudreau, D. Meintel, L.M. Dubois, N. Labrie et P. Lamarre, CRSH

«Transmission culturelle et identitaire chez les jeunes couples mixtes », D. Meintel, CRSH 